\title{
The active guidewire technique versus standard technique as different way to approach ureteral endoscopic stone treatment
}

\author{
Alessandro Calarco ${ }^{1}$, Marco Frisenda ${ }^{1,3}$, Emilio Molinaro ${ }^{1,3}$, Niccolò Lenci $^{2}$ \\ ${ }^{1}$ Department of Urology, "Cristo Re" Hospital Fondation, Rome, Italy; \\ 2 Department of Urology, "A. Gemelli” Academic Hospital, Catholic University of Sacred Heart, Rome, Italy; \\ ${ }^{3}$ Department of Urology, Policlinico Umberto I, "La Sapienza" University, Rome, Italy.
}

\begin{abstract}
Summary Background: One of the greatest challenges in semi-rigid ureteroscopies, for both stones and tumors, is the control of endoscopic vision and the maintenance of low intracavitary liquid pressure. We present a comparison between two operative techniques: in the first method an ordinary guide wire (diameter 0.032") is used for the procedure; in the second one a $5 \mathrm{Fr}$ ureteral catheter replaces the guidewire (we called it "Active guidewire")

Methods We compared 50 semirigid ureteroscopies (sURS) performed using the active guidewire with another 50 procedures conducted with a classic guidewire. We evaluated the difference in operating times, quality of endoscopic vision, periprocedural infections rate and stone-free rate.

Results: The use of active guidewire has considerably reduced the standardized operating times per unit stone-volume by about $39 \%$. Vision quality has improved considerably thanks to the continuous flow in-and-out. Consequently, periprocedural infections decreased (3\% vs 30\%) and the stone-free rate rose from $86 \%$ to $92 \%$.

Discussion and conclusions: Employing an "active guidewire" instead of the standard guidewire, the risk of complications related to high pressures and operating time is considerably lower, as well as better treatment quality thanks to the cleaner vision. This technique has proven to be safe as well as easy to apply, and in our belief is to be preferred whenever the ureter accepts without forcing, both the presence of the catheter and the semi-rigid $7 \mathrm{~F}$ ureteroscope.
\end{abstract}

KEY WORDS: Ureteroscopy; Ureter; Guidewire; Ureteral stones; Retrograde intrarenal surgery; Lithotripsy.

Submitted 12 July 2021; Accepted 25 August 2021

\section{INTRODUCTION AND BACKGROUND}

In ureteral pathologies where indication is endoscopic treatment such as stones or urothelial tumors, the growing technological progress has led to a more frequent use of minimally invasive operative techniques including semi-rigid ureteroscopy and RIRS (Retrograde IntraRenal Surgery). All of these operating techniques use irrigation to permit the best possible endoscopic vision and simple safety maneuvers to minimize the risk of complications. Advances in technology have also generated ureterorenoscopes with increasingly smaller diameters, but the price to pay for this reduction in diameter is one common channel for both irrigation and accessories, resulting in decreased irrigation flow. Good irrigation means good vision (1), poor irrigation means poor vision. It is clear that the key factor determining the best outcome of the intervention is based on the quality of the vision with containment of complications in association with reasonable operating times.

In our belief, the most important safety maneuvers are the positioning of a safety guidewire, the use of low pressures and avoiding to force the advancement of the instrument. During semirigid ureteroscopy, whether it is for stones or for cancer, one of the essential conditions is certainly the quality of vision that can be implemented by better tools like digital cameras but remains very conditioned by the quality of the medium in which the camera is immersed. Moreover, there are concerns about the changes in the intra-pelvic pressure (IPP) that might reach critical levels, resulting in pyelovenous, pyelolymphatic, and pyelointerstitial backflow with subsequent systemic inflammatory response syndrome and sepsis.

The association between surgery time and post-operative infectious complications was discussed in several studies in literature involving $>7.000$ patients. According to the primary overall meta-analysis result, patients with longer operation time ( $\mathrm{min}$ ) are more vulnerable for infectious complication $(\mathrm{OR}=1.03,95 \% \mathrm{CI}: 1.01-1.04, \mathrm{I} 2=70.6 \%$, $\mathrm{p}=0.001)$. According to the subgroup analysis, patients with longer operation time (min) are vulnerable or postoperative fever/urinary tract infections $(\mathrm{OR}=1.02,95 \%$ CI:1.01-1.03, p < 0.001, I2 = 15.2\%, p = 0.308) and also vulnerable for post-operative systemic inflammatory response syndrome/urosepsis (OR:1.08, 95\% CI 1.02$1.14, \mathrm{p}=0.009, \mathrm{I} 2=69.4 \%, \mathrm{p}=0.0017)(2,3)$.

The rationale of our study is to make a comparison between endoscopic procedures performed with the use of a standard guidewire and a ureteral catheter, which allow to create a continuous flow, so that the intake liquid is given by the instrument and the outflow by the ureteral catheter. That variation generates a potentially nonstop laser procedure with reduced operating-time, better vision, and a decreased risk of high Intra Pelvic Pressure. This procedure can be called "active guidewire" technique, because of the active role of the ureteral catheter.

No conflict of interest declared. 


\section{Methods}

To overcome the problems of the current technique, such as poor vision and interruption of the flow at high intrarenal pressures, we describe the differences between the two techniques and in particular the advantages seen in the population treated with the active guidewire.

In this technique the safety guidewire is replaced by a Pollack ureteral catheter (COOK Medical ${ }^{\circledR}$ ) thus becoming an active, and no longer passive, element during the procedure.

The "active guidewire" has a diameter of $5 \mathrm{~F}$, with a soft tip at its head, and it is positioned in place of the safety guidewire, thus connecting the renal pelvis with the outside. The Pollack catheter has an internal size of $4 \mathrm{Fr}$. A $6,5 / 7 \mathrm{~F} \mathrm{Storz}^{\circledR}$ semirigid ureteroscope is used and it has a $4 \mathrm{~F}$ irrigation channel. The two $4 \mathrm{~F}$ channels give us a perfect balance with in and out irrigation flow.

A polytetrafluoroethylene (PTFE)-coated guidewire was used as standard guidewire. A PTFE-Nitinol guidewire with hydrophilic tip was used when the stone was impacted.

For statistical evaluation IBM $^{\odot}$ SPSS Statistics program (Illinois, Chicago, v. 24) was used.

Pearson's Chi-Square, Pearson correlation analysis and Student's test were used. Statistical significance was evaluated at $\mathrm{p}<0.05$.

We took in consideration 100 patients undergoing semirigid ureterorenoscopy (sURS) for ureteral stones. Hydronephrosis was present in $60 \%$ of the patients. No double J (JJ) stent was inserted before the surgery, in any case (Table 1).

All patients had a clinical evaluation, urine dipstick analysis with additional culture and sensitivity testing if a urinary tract infection (UTI) was suspected, a measurement of serum creatinine level, abdominal ultrasonography (US) and a plain abdominal X-ray.

Patients with positive pre-operative urine culture (n. 4) were treated with specific antibiotics until complete remission (verified performing new urine culture and blood test). Preoperative additional computed tomography (CT) was used, according to the level of serum creatinine and stone radiolucency, in that patients in which US and $\mathrm{X}$-ray were not adequate for the diagnosis. Patients were placed in the lithotomy position and received prophylactic parenteral antibiotics before the procedure, which was performed under spinal or general anesthesia. A retrograde pyelogram was performed to define the anatomy and visualize any filling defect.

We created two groups. Group A: 50 patients underwent "active guidewire" technique and 50 underwent standard technique. The ureteral catheter was used until the end of the procedure in all patients of group A instead of the standard guidewire. Group B: 50 patients underwent standard procedure with the aid of traditional guidewire. The initial phases of both the surgical procedures were similar, up to the step of replacing the standard guidewire with the active one.

Firstly, using a cystoscope, a urethrocystoscopy was performed to exclude any other urethral or bladder pathologies. Then a PTFE standard guidewire was placed in the ureter and the cystoscope removed. A second guidewire through the operative channel of $7 \mathrm{~F}$ ureteroscope was used to reach the stone, passing the instrument between the two guidewire. If the ureter appeared compliant and the stone was not completely obstructing the lumen, we removed the ureteroscope and we replaced the standard guidewire with a 5 F Pollack catheter, left aside the stone until the end of the procedure. This step was performed with the help of the fluoroscopy and contrast enhancement. Finally, the ureteroscope was reintroduced to begin the lithotripsy.

Holmium-YAG laser was used for stone dusting or fragmentation. Operating time was considered from the beginning of the procedure (operator introduced the guidewire in the ureteral meatus) to the end (operator placed the ureteral stent).

We evaluated as endpoints:

- reduction of operating times related to the volume of the stone.

- quality of endoscopic view (expert operator opinion $\mathrm{AC})$.

- reduction of the number of procedure-related Urinary Tract Infections (UTIs) by monitoring the leukocytes blood values before and after surgery.

- stone free rate (SFR) valuated by 30 days no-CE CT scan.

All procedures were performed by the same operator in high volume center with the same type of semi-rigid ureteroscope. Patient's data collected, including age, gender, side, stone location (proximal, intermediate or distal ureteral).

\section{RESULTS}

We observed a statistically significant reduction ( $p=$ 0.01 ) in operating times of the procedure performed with the "active guidewire" compared to the control group, standardizing the operating times and relating them to the volume of the stone. We calculated a coefficient given by the ratio between the operating time ( $\mathrm{min}$ ) and the volume of the stone $\left(\mathrm{mm}^{3}\right)$.

This coefficient was 39\% lower in the procedures conducted with active guidewire (5.72 vs 9.40) (Table 2). Pearson correlation analisys was conducted, that demostrates a direct correlation in favour of the active guidewire technique $(\mathrm{r}=0.208, \mathrm{p}=0.035)$ (Table 3$)$.

In 10 patients of group $\mathrm{A}$ we found a reduction in post-

Table 1.

Patient demographics.

\begin{tabular}{|l|c|}
\hline Patient, $n$ & 100 \\
\hline Mean agge years (range, median) & $54(28-86,56)$ \\
\hline Men, $n(\%)$ & $63(63)$ \\
\hline Women, $n(\%)$ & $37(37)$ \\
\hline Proximal ureteral stone, $n(\%)$ & $32(32)$ \\
\hline Intermediate ureteral stone, $n(\%)$ & $26(26)$ \\
\hline Distal ureteral stone, $n(\%)$ & $42(42)$ \\
\hline Hydronephrosis, $n(\%)$ & $78(78)$ \\
\hline Side right/left (\%) & $49 / 51(49 / 51)$ \\
\hline Previous RIRS (\%) & $0(0)$ \\
\hline Indwelling double-J stent, (\%) & $0(0)$ \\
\hline
\end{tabular}


Table 2.

Data analysis.

\begin{tabular}{|l|c|c|c|}
\hline & "Active guidewire" & Standard guidewire & P-value \\
\hline Completed procedures sURS (\%) & $50(50)$ & $50(50)$ & - \\
\hline Average age & 55 & 53 & - \\
\hline $\begin{array}{l}\text { Average ratio operating time|stone } \\
\text { volume (min }\left(\mathrm{mm}^{3}\right)\end{array}$ & 5.72 & 9.4 & 0.01 \\
\hline $\begin{array}{l}\text { Mean increased postoperative } \\
\text { leukocytosis in percentage }\end{array}$ & 3 & 30 & $<0.01$ \\
\hline $\begin{array}{l}\text { Mucosal injury (\%) } \\
\text { Postoperative fever (\%) }\end{array}$ & $0(0)$ & $5(10)$ & $<0.01$ \\
\hline Stone-free rate (\%) & $46(92)$ & $8(16)$ & $<0.01$ \\
\hline
\end{tabular}

Table 3.

Pearson correlation analysis.

\begin{tabular}{|l|c|c}
\hline Variable & Person coefficent & P-value \\
\hline Endoscopic technique - operating time & -0.532 & $<0.001$ \\
\hline Endoscopic technique - postoperative leukocytosis & -0.654 & $<0.001$ \\
\hline Endoscopic technique - mucosal slippage & -0.246 & 0.012 \\
\hline Endoscopic technique - operating time $\mid$ stone volume $\left(\mathrm{min} \backslash \mathrm{mm}^{3}\right)$ & 0.208 & 0.035 \\
\hline
\end{tabular}

operative leukocytosis, thanks to the resolution of hydronephrosis. In the remaining 40 patients of group A we witnessed a postprocedural increase of the white blood cell count $(<20 \%)$. The mean increase of white blood cell count across all procedures performed with active guidewire was 3\% compared to pre-operative blood cell count. Two of these patients had postoperative fever and leukocytosis $>20.000 \mathrm{WBC} / \mathrm{mm}^{3}$, treated with antibiotic therapy. All patients in group B showed rising

Figure 1.

Clear vision of operative area with the use of an active guidewire.

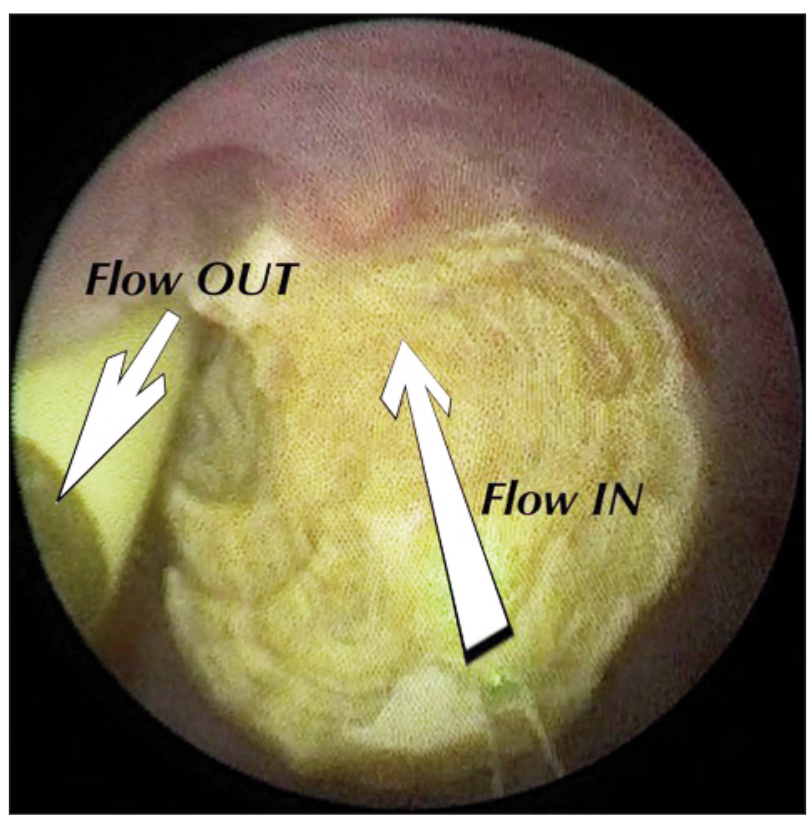

white blood cell count, with an average white blood cell growth of $30 \%$. Statistical analysis demonstrates a significative difference in reduction of leukocytosis, number of mucosal slippage and post-operative fever (Table 2).

In group B, 5 episodes of ureteral mucosal injury occurred, which led to an early conclusion of the procedure and the placing of a ureteral stent.

Stone free rate (SFR) was $92 \%$ and $86 \%$ respectively in "active guidewire" and standard guidewire group. No significant differences occurred on this field (Table 2).

Pearson correlation analysis demonstrated an indirect correlation between the endoscopic technique and operating time $(r=-0.532, p<0.001)$, mucosal slippages $(r=-0.246$, $\mathrm{p}=0.012)$ and postoperative leukocytosis $(\mathrm{r}=-0.654$, $\mathrm{p}<0.001$ ) (Table 3).

\section{Discussion}

A compliant ureter is defined as a ureter $\geq 12 \mathrm{~F}$ and so it should allow the easy passage of a semirigid $7 \mathrm{~F}$ ureteroscope with a safety guidewire (3 F) aside or "active guidewire" (5 F) (4). The usage of a ureteral catheter instead of the standard guidewire has some remarkable advantages. First of all, the quality of vision, in particular during laser lithotripsy, is absolutely better than the standard technique because the generated powder is immediately expelled through the catheter, thanks to the constant antegrade flow. Stone powder is not in suspension in our working area. The second advantage, directly connected to the first, is represented by a lower risk of ureteral mucosal injury due to a better vision (Figure 1).

In group $\mathrm{B}$, we observed 5 episodes of ureteral mucosal injury, occurred during laser lithotripsy. The cause of these injuries was the imperfect vision of the operating field, due to the stone powder and the necessity of a reduced or discontinuous inflow, in order to not push-up the stone. In fact, no ureteral tears or damage occurred in group A, because of a perfect vision of the ureteral field. Finally, it allows to reduce intrarenal pressure peaks. During the semi-rigid ureteroscope progression in ureter, the IPPmax (intrapelvic pressure Max) reaches high levels in renal pelvis; the use of pumpes also induces critical levels of IPPmax. Excessive irrigation pressures can be detrimental, leading to pyelolymphatic and pyelovenous backflow and consequent development of sepsis $(1,5)$.

The use of a Pollack ureteral catheter during ureteroscopy, allow an excape way for saline irrigation preventing high pressure peaks. We were not able to evaluate the real renal pelvis IPP ${ }_{\max }$ in the two groups, but we suppose that the presence of the $5 \mathrm{~F}$ ureteral catheter in pelvis can create a useful way of outflow. We think that the lower number of post-operative leukocytosis and fevers in Group A could be due to this expedient.

In summary, a better vision of the operative area, the reduction of operating time and the low rate of leukocytosis can traslate in better results for this kind of surgery. In addition, during semi-rigid ureteroscopy it is mandatory to interrupt the treatment every time the antegrade flow ceases, due to the filling of the excretory route, because of the absence of a continuous flow of the instrument. So the usage of the ureteral catheter reduces the IPPmax, post-operative UTIs and allows a faster hospital 
discharge of the patient (6-8). It results in a decrease of hospitalization costs.

\section{Conclusions}

If we consider the kidney as a closed system, without a discharge for saline irrigation during semi-rigid ureteroscopy, we have to consider that the most part of time is actively used (antegrade flow, good vision and lithotripsy) but a really long period of time is spent passively (discharge of the kidney), so it leads to a temporary interruption of the procedure.

In our experience the "active guidewire technique" has proven to be safe as well as easy to apply in selected cases. The use of a small semi-rigid ureteroscope $(7 \mathrm{~F})$ is mandatory, because of the $5 \mathrm{~F}$ diameter of the ureteral catheter. We believe that this technique, compared to the use of the traditional guide wire, is to be preferred whenever the ureter allows the presence of the catheter and the semirigid $7 \mathrm{~F}$ ureteroscope (for a total of $12 \mathrm{~F}$ ) in its lumen, avoiding forcing the passage of the instrument.

The size of the catheter is acceptable, in consideration of the frequent use of ureteral sheaths with even larger diameters (from 12 to $14 \mathrm{Fr}$ ) in endoscopic urological surgery (9). The decompression of the pelvic-caliceal system leads to avoid pressure spikes, which might decrease infectious complications. Furthermore, the risk of complications related to high pressures and operating time is lower, as well as better treatment quality thanks to cleaner vision (10-12).

Many authors suggested that high intrarenal pressure during ureteroscopy and iatrogenic trauma of the pelvicalyceal system during instrumental manipulations are the most probable mechanisms that lead to other life-threatening complications such as urinomas, perirenal abscesses and subcapsular, perirenal and retroperitoneal hematoma, reported with an incidence up to $2,2 \%(13,14,16,17)$.

Nevertheless, the use of "active guidewire" can decrease the IPPmax and let URS safer by acting as a safeguard against the consequences of increased IPP, even under manual pumping and forced irrigation (15). It needs a laboratory study to assess the real value of the IPP and the differences in the two surgical approaches.

The comparison also needs a larger cohort of study to be better statistically evaluated in order to standardize a different and often safer approach to ureteral and renal stone.

\section{ETHICS APPROVAL AND CONSENT TO PARTICIPATE}

All procedures performed in studies involving human participants were in accordance with the ethical standards of the institutional research committee and with the 1964 Helsinki declaration and its later amendments or comparable ethical standards. Each patient agreed to allow the use of their clinical data for the study and signed an informed consent.

This study doesn't require the approval by the ethics committee for its conception.

\section{AvaILABILITY OF DATA AND MATERIALS}

The data that support the findings of this study are available on request from the corresponding author AC.
The data are not publicly available for containing personal information that could compromise research participant privacy.

\section{FUNDING}

This study was not funded by any institution.

Author contributions (initials name and surname):

(I) Conception and design: AC, NL

(II) Administrative support: MF, EM

(III) Provision of study materials or patients: AC

(IV) Collection and assembly of data: AC. NL

(V) Data analysis and interpretation: NL, MF, EM

(VI) Manuscript writing: All authors

(VII) Final approval of manuscript: All authors.

\section{REFERENCES}

1. Michel MS, Honeck P, Alken P. Conventional high pressure versus newly developed continuous-flow ureterorenoscope: urodynamic pressure evaluation of the renal pelvis and flow capacity. J Endourol. 2008; 22:1083-1085.

2. Ma YC, Jian ZY, Yuan C, et al. Risk factors of infectious complications after ureteroscopy: a systematic review and meta-analysis based on adjusted effect estimate, Surg Infect. 2020; 21:811-822.

3. Southern JB, Higgins AM, Young AJ, et al. Risk Factors for postoperative fever and systemic inflammatory response syndrome after ureteroscopy for stone disease. J Endourol. 2019; 33:516-522.

4. Fulla J, Prasanchaimontri P, Rizk A, Loftus C, et al. Ureteral diameter as predictor of ureteral injury during ureteral access sheath placement. J Urol. 2021; 205:159-164.

5. Li T, Sun XZ, Lai DH, et al. Fever and systemic inflammatory response syndrome after retrograde intrarenal surgery: Risk factors and predictive model. Kaohsiung. J Med Sci. 2018; 34:400-408.

6. Moses RA, Ghali FM, Pais VM, Jr, Hyams E. Unplanned hospital return for infection following ureteroscopy - Can we identify modifiable risk factors? J Urol. 2016; 195:931-936.

7. Mitsuzuka K, Nakano O, Takahashi N, Satoh M. Identification of factors associted with postoperative febrile urinary tract infection after ureteroscopy for urinary stones. Urolithiasis. 2016; 44:257262.

8. Blackmur JP, Maitra NU, Marri RR, et al. Analysis of factors association with risk of postoperative urosepsis in patients undergoing ureteroscopy for treatment of stone disease. J Endourol. 2016; 30:963-969.

9. Boulalas I, De Dominicis M, Defidio L. Semirigid ureteroscopy prior retrograde intrarenal surgery (RIRS) helps to select the right ureteral access sheath. Arch Ital Urol Androl. 2018; 90:20-24.

10. Ogreden E, Oguz U, Demirelli E, et al. Categorization of ureterooscopy complications and investigation of associated factors by using the modified Clavine classification system. Turk J Med Sci. 2016; 46:686-694.

11. Somani BK, Giusti G, Sun Y, et al. Complications associated with ureterorenoscopy (URS) related to treatment of urolithiasis: the clinical research Office of Endourological Society URS Global study. World J Urol. 2017; 166:538-540.

12. Proietti S, Dragos L, Somani BK, Buttice S, Talso M, Emliani E, et al. In vitro comparision of maximum pressure developed by irrigation systems in a kidney model. J Endourol. 2017;31:522-527. 
13. De Coninck V, Keller EX, Somani B, et al. Complications of ureteroscopy: a complete overview. World J Urol. 2020; 38:21472166.

14. Bai J, Li C, Wang S, et al. Subcapsular renal haematoma after holmium: yttrium-aluminium-garnet laser ureterolithotripsy. BJU Int. 2012; 109:1230-1234.

15. Hyams ES, Munver R, Bird VG, et al. Flexible ureterorenoscopy and holmium laser lithotripsy for the management of renal stone bur- dens that measure 2 to $3 \mathrm{~cm}$ : a multi-institutional experience J Endourol. 2010; 24:1583-1588.

16. Xu L, LiG Life-threatening subcapsular renal hematoma after flexible ureteroscopic laser lithotripsy: treatment with superselective renal arterial embolization. Urolithiasis. 2013; 41:449-451.

17. Meng HZ, Chen SW, Chen GM, et al. Renal subcapsular Hemorrhage complicating ureterolithotripsy: an unknown complication of a know day-to-day procedure. Urol Int. 2013; 91:335-339.

\section{Correspondence}

Alessandro Calarco, MD

alecalarco@gmail.com

Marco Frisenda, MD (Corresponding Author)

marco.frisenda57hu@gmail.com

Emilio Molinaro, MD

emilio.molinaro89@gmail.com

Department of Urology, "Cristo Re" Hospital Fondation, Rome (Italy)

Niccolò Lenci, MD

lenci.niccolo@live.com

Department of Urology, "A. Gemelli” Academic Hospital, Catholic University of Sacred Hearth, Rome, Italy 